\title{
PANDORA'S BOX
}

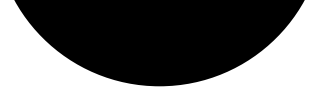

Pandora searches the world literature for evidence, news and other sources on matters of interest (doesn't shy away from controversy) to bring to the reader. She welcomes comments and suggestions (via ip@rcpsych. ac.uk)

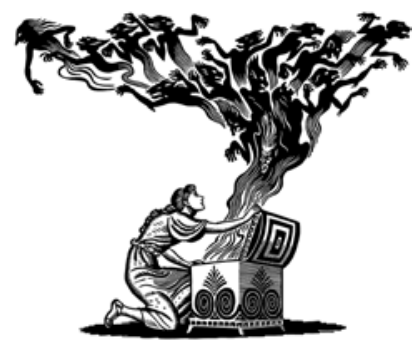

Food for thought - the Mediterranean diet (MeDi) is good for your brain!

1 he Mediterranean diet, high in vegetables and fish, is recognised not only for its beneficial effects on our general physical health but also for reducing the risk for cognitive impairment. But does it have any measurable effects on brain structure? In a cross-sectional study of older people (mean age 79.8 years), male and female, with normal cognitive function, those on a diet high in vegetables and fish had thicker cortices in the frontal, parietal and occipital lobes. However, a word of caution for those with a 'sweet tooth'. The study also found a decrease in the thickness of the entorhinal cortex in those who indulged in higher carbohydrate intake.

It would be interesting to see if prospective studies confirm all these findings but in the meantime Pandora will stick to MeDi and go easy on sugars!

Staubo, S. C., Aakre, J. A., Vemuri, P., et al (2016) Mediterranean diet, micronutrients and macronutrients, and MRI measures of cortical thickness. Alzheimer's and Dementia. doi: 10.1016/j. jalz.2016.06.2359. [Epub ahead of print]

\section{Turning off expression of the Huntington disease gene}

T he team running the FINGERS4CURE project, which is funded by the European Union and led by researchers at Imperial College London, have engineered a 'zinc finger', a protein targeting the huntingtin (HTT) gene, found in people with Huntington's disease, and repressing its ability to create toxic levels of harmful proteins in the brain.

Although the precise cause of the disease is still not known, it is hoped that turning off that gene's expression could be beneficial. The results from a trial in mice are encouraging. A single injection of the therapeutic protein reduced the activity of the gene for several months. Could there finally be a prophylactic treatment for this devastating condition?

Agustín-Pavón, C. Mielcarek, M., Garriga-Canut, M., et al (2016) Deimmunization for gene therapy: host matching of synthetic zinc finger constructs enables long-term mutant Huntingtin repression in mice. Molecular Neurodegeneration. doi:10.1186/s13024-016. 0128-x

\section{Keep your toddler's brain active!}

We can't retrieve memories of events that took place during our first 2-4 years of life, but do they matter? According to scientists, these infantile memories, which we cannot remember (infantile/ childhood amnesia), are important to our brain development. Experiments in rats show that early life experiences, although not remembered, can influence adult life behaviour.

This is a critical period for episodic learning, when the hippocampus becomes able to efficiently process and store memories in the long term, and which concerns important functions such as vision and language acquisition.

In a series of experiments it was found that if the hippocampus is inactive during this developmental stage, when the nervous system is especially sensitive to environmental stimuli, such latent memories formed could not be recalled in later life by reminders.

Essentially, during this critical period of early life, the brain learns how to form long-term memories. The brain needs stimulation to enable it to achieve this; if it fails to do so, the developmental ability of the neurological system is impaired. The authors suggest that using learning and environmental interventions during this critical period may help significantly in addressing intellectual disabilities.

Travaglia, A., Bisaz, R., Sweet, E. S., et al (2016) Infantile amnesia reflects a developmental critical period for hippocampal learning. Nature Neuroscience. doi: 10.1038/nn.4348. [Epub ahead of print] PMID:27428652

\section{Antisocial behaviour - brain maldevelopment or teenage rebellion?}

Teuroimaging that investigates structural covariance between brain regions is an increasingly used approach to investigating the synchronised maturation of different brain regions and the study of psychiatric disorders with presumed neurodevelopmental origins.

Researchers from the universities of Cambridge, Southampton and Rome used magnetic resonance imaging (MRI) to examine brain structure in males with a diagnosis of conduct disorder and healthy controls, aged 16-21 years. They calculated interregional correlations in cortical thickness as a measure of structural covariance in male youths with childhood-onset conduct disorder (CO-CD), adolescence-onset CD (AO-CD) and healthy controls, in two independent patient samples.

The CO-CD youths displayed more significant interregional correlations than the $\mathrm{AO}-\mathrm{CD}$ youths and the healthy controls, whereas the AO-CD individuals displayed fewer correlations than the healthy controls. The three groups differed in the strength of the interregional correlations across frontal, temporal, parietal and occipital regions.

The researchers' interpretation of the results is that they reflect specific disruptions in the development of the brain during adolescence. They concluded that this is compelling evidence that conduct disorder is a 'real psychiatric disorder' and not an 'exaggerated form of teenage rebellion'. Further research is needed to examine the factors leading to this abnormal pattern of brain development, such as exposure to early adversity, and investigate further how to use these results to help young people with conduct disorder.

Fairchild, G., Toschi, N., Sully, K., et al (2016) Mapping the structural organization of the brain in conduct disorder: replication of findings in two independent samples. Journal of Child Psychology and Psychiatry. doi: 10.1111/jcpp.12581

\section{Misuse of prescription drugs in the European Union}

T is known that prescription drugs are passed 1 around families and friends and in some cases there is also a commercial element! Until recently 
this was thought to be mostly a problem in the United States, where the lifetime rate of prescription drug misuse, for those aged 12 years or more, is as high as $20 \%$. However, a recent study shows that the European Union is not far behind, with lifetime rates between $7 \%$ and $13 \%$ for substances such as opioid analgesics. The European Union Medicine Study collected the data from a population of 20000 adults and 2000 teenagers. Spain, Sweden and the UK have high rates of misuse, while Germany has the lowest rates. Interestingly, the internet was the least common way of getting hold of prescription drugs.

Among those misusing prescription drugs there were high rates of poly-drug use: $52 \%$ of those prescribed stimulants, $32 \%$ of prescribed opioid users and $28 \%$ of those on prescribed sedatives also used illicit drugs.

This is the first comparative study of prescription drug misuse in the European Union and it raises an alarm about a problem that may increase if no appropriate measures are taken.

Novak, S. P., Håkansson, A., Martinez-Raga, J., et al (2016) Nonmedical use of prescription drugs in the European Union. BMC Psychiatry. doi: 10.1186/s12888-016-0909-3.

\section{Is life precious enough for all?}

World Suicide Prevention Day was 10 SepVtember 2016. It was organised by the International Association for Suicide Prevention (IASP) and the World Health Organization (WHO). This serves as a reminder for all those involved with people at suicide risk to up their act.

According to the WHO, every 40 seconds one person somewhere in the globe puts an end to his or her life: a total of 800000 people every year. Globally, suicide rates have been increasing.

The 25 countries with the highest suicide rates differ significantly from each other in a variety of ways, including geographical position, culture and socioeconomic conditions. Almost all continents are represented and men outnumber women in all countries by a factor of 2-3 or more in many cases. The countries with the highest suicide rates are, in ascending order, Poland at 16.6 per 100000 (30.5 among men; 3.8 among women), Ukraine, Sudan, Comoros, Bhutan, Zimbabwe, Belarus, Japan, Hungary, Uganda, Russian Federation, South Sudan, Turkmenistan, Burundi, Kazakhstan, Nepal, Tanzania, Mozambique, Surinam, Lithuania, Sri Lanka, South Korea and North Korea. The South American country Guyana tops the list with a suicide rates of 44.2 per 100000 (70.8 among men; 22.1 among women). Perhaps the adequacy of the methodology in different countries may be not as high as would be desirable, and the resulting data may be questioned for accuracy, but the figures cannot be completely dismissed. There is a pattern that cannot be ignored.

Although suicide is in our domain when due to mental illness and there is no question that, as psychiatrists, we should be doing our utmost to reduce suicide rates, can we really make a big difference globally? Aren't conflict, poverty, oppression and dramatic political and economic changes that turn people's lives upside down at the root of many suicides, or, dare I say, mostly responsible for people opting for death? Shouldn't the politicians and primarily the world leaders be discussing such matters? Should it be on the G20 agenda?

World Health Organization. Suicide Rates Data By Country. WHO, 2012.

\section{Enjoy your coffee - it is good for you!}

Tnlike other stimulants, coffee has never been found to be associated with any harm to human beings. A recent meta-analysis to assess the influence of coffee drinking on the risk of developing cognitive disorders is more than reassuring for us coffee addicts! Nine prospective cohort studies involving 34282 participants were included, with follow-up ranging from 1.3 to 28 years. Daily drinking of one or two cups of coffee was associated with a lower occurrence of cognitive disorders, including Alzheimer's disease.

Well you now know that coffee is good for your brain, but did you know that it is also good for your liver? Evidence shows that it reduces liver enzymes in viral hepatitis, non-alcoholic fatty liver disease (NAFLD) and cirrhosis, and it also offers some protection from hepatocellular carcinoma. However, how your coffee is made matters. It appears that filter coffee may be best for you, rather than espresso and barista. It filters out some bad-for-you substances such as kahweol and cafestol, which are caffeine diterpenes, released from ground coffee beans but removed by the paper filters.

The bad news for tea drinkers and those favouring other caffeine drinks is that they don't have the benefits that coffee offers; it seems that substances in addition to caffeine, contained in coffee, are also needed.

Wu, L., Sun, D. \& He, Y. (2016) Coffee intake and the incident risk of cognitive disorders: a dose-response meta-analysis of nine prospective cohort studies. Clinical Nutrition [Epub ahead of print]. doi: 10.1016/j.clnu.2016.05.015. PMID: 27288328

Saab, S., Mallam, D., Cox, II, J. A., et al (2014) Impact of coffee on liver diseases. A systematic review. Liver International, 34 (4), 495-504.

\section{E-cigarettes - to use or not to use?}

$\mathrm{f}$, as you are enjoying your coffee, you get tempted to light a cigarette, remind yourself that, unlike coffee, cigarettes are proven to cause serious harm! What about e-cigarettes? There are concerns that e-cigarettes' increasing popularity may be undermining successful quitting and this would have an adverse effect on public health. A recent publication in the $B M J$ examined the prevalence of e-cigarette use in current smokers and during a quit attempt, to predict quit success. Don't throw your e-cigarette away! The authors found that changes in the prevalence of e-cigarette use in England have been positively associated with the success rates of quit attempts.

Beard, E., West, R., Michie, S., et al (2016) Association between electronic cigarette use and changes in quit attempts, success of quit attempts, use of smoking cessation pharmacotherapy, and use of stop smoking services in England: time series analysis of population trends. BMJ, 354, i4645. doi: http://dx.doi.org/10.1136/bmj.i4645. 